\title{
Dynamic Instability of Strongly Confined Thin Polymer Films in Spinodal Dewetting
}

\author{
K. Y. Suh and Hong H. Lee* \\ School of Chemical Engineering, Seoul National University, Seoul, 151-742 Korea
}

(Received 4 April 2001; published 7 September 2001)

\begin{abstract}
We present a general criterion for determining the dynamic instability of a thin polymer film that is strongly confined to a substrate. When such a polymer film is heated above its glass transition temperature, it dewets from the substrate by a spinodal dewetting scenario, in which the onset of the instability is governed by dispersion force and residual thermal stress. It turns out that the thermal stress plays an important role when there is a special interaction between a polymer and a substrate.
\end{abstract}

DOI: $10.1103 /$ PhysRevLett.87.135502

PACS numbers: 61.41.+e, 68.08.Bc, 68.15.+e

Thermal stability of thin films in the dewetting process has drawn much interest in recent years [1-5]. Because of technological importance and a convenient experimental time scale, most experiments have been devoted to the instability of thin polymer films [6-9]. In particular, a low molecular weight polymer $\left(M_{w}<10^{4}\right)$ has frequently been used for its liquidlike behavior above the glass transition temperature [6,7]. This liquidlike behavior of the polymer is well matched with the conventional capillary wave theory such that the cutoff wavelength and dynamic instability can successfully be explained [7].

Recently, several experiments have been reported on the dewetting behavior of a high molecular weight polymer and metal films [8-10]. In contrast to what is widely believed, the dewetting behavior is quite different from what is expected from conventional theory. The capillary wave theory predicts that the cutoff wavelength $(\lambda)$ is related to the film thickness $(h)$ as $\lambda \propto h^{2}$ [3]. Wang et al. [8] reported, however, that the power index is not 2 but rather ranges from 0.8 to 1 for high molecular weight polyvinyl-pyridine (PVP) and polystyrene (PS) $\left(M_{W}=\right.$ $9 \times 10^{4}$ ) thin films. As PVP and PS strongly react with $\mathrm{SiO}_{2}$ and $\mathrm{Si}$ substrates, respectively, they attributed this anomalous behavior to solidlike or viscoelastic properties of the polymers. Tolan et al. [9] also reported that the power index is about 1.6 for high molecular weight polyethylene-propylene $\left(M_{w}=2.9 \times 10^{5}\right)$ thin films. For the metal films, Bischof et al. [10] reported that the power index is as low as 1.3 with film thickness ranging from 25 to $50 \mathrm{~nm}$, which is much smaller than the value predicted from the capillary wave model.

In general, the thermal stability of an initially stressed solid film is determined by the competition between elastic energy and surface energy. In this case, the elastic energy is the destabilizing factor, which facilitates the pattern formation, whereas the surface energy inhibits the surface roughening. Extensive studies have been devoted to clarifying the surface patterning of semiconductor or ceramic films [11]. As is well known, films should be rather stiff for the destabilizing elastic force to come into play.

On the other hand, an initially stress-free solid film is thermally stable unless there is a special interaction at the free surface caused by an external force such as an electrical field [12]. This is readily understood in that both elastic and surface energies contribute to the stabilization. In the case of a special interaction at the free surface, however, it was reported that instability sets in and the corresponding wavelength is always of the same order as the film thickness and scales linearly with it [13].

In contrast to solid films, thermal stability of liquid films is well described by the destabilizing long-range van der Waals interaction (i.e., Hamaker constant is positive) and the stabilizing surface energy [3]. Unless there is a special interaction at the polymer-substrate interface, the polymer can be assumed to be a viscous liquid and the thermal stress accumulated by the temperature rise may easily be dissipated above the glass transition temperature $\left(T_{g}\right)$. However, the thermal stress may not be annihilated if there is a strong interaction at the film-substrate interface. Wallace et al. [14] suggested that strongly confined thin polymer films consist of two layers (Fig. 1) in systems such as PS on hydrogen-terminated $\mathrm{Si}$ and PVP on $\mathrm{SiO}_{2}$. Note in this regard that strong confinement means special interactions at the film-substrate interface. In this case, an ultrathin layer forms at the polymer-substrate interface, which shows no glass transition much above the bulk glass transition temperature. Furthermore, the average thermal expansion coefficient of the film is smaller than that of the bulk both below and above $T_{g}$, which indicates strong confinement to a substrate. Ordinarily, the magnitude of thermal

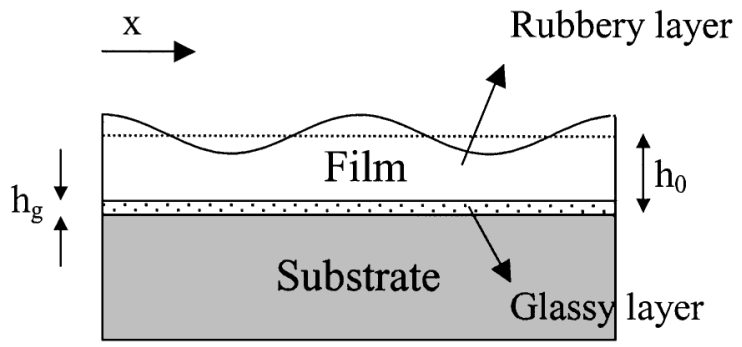

FIG. 1. Thin polymer film that is strongly confined to a substrate. Because of the strong confinement at the polymersubstrate interface, glassy and rubbery layers are present even above $T_{g}$. 
stress drops by 3-4 orders in magnitude when a polymer film undergoes a glass-rubber transition, giving negligible thermal stress above $T_{g}$. On the other hand, a confined thin layer modifies the overall film properties to a rather solidlike behavior, which helps maintain the stress. In particular, the built-in stress may not be easily dissipated if the entanglement effect takes hold (i.e., $M_{w}>4 \times 10^{4}$ for PS) [15] and the movement is quite sluggish.

In this Letter, we present a general model that can explain the dynamic instability of thin polymer films. We propose that the thermal stress together with the dispersion force be included in the spinodal dewetting model to completely explain the dewetting behavior.

The system being considered is illustrated in Fig. 1. We designate the lateral direction in which the spinodal wave propagates as $x$. The equation of motion for the lateral flow in a thin film can be written from the continuity equation as follows [16]:

$$
\frac{\partial}{\partial t} h(x, t)=C \frac{\partial^{2}}{\partial x^{2}} p[h(x, t)],
$$

where $h(x, t)$ is the local film thickness, $C$ is the constant that is responsible for the shape of the flow profile and the viscosity of the film, and $p(x, t)$ is the film pressure.

Because of the strong interaction between the polymer repeat unit and the substrate surface, an ultrathin glassy layer forms at the interface. This can be understood as cross-linking of polymer chains close to a substrate where it inhibits the chains from attaining the entropy necessary to reach the melt state. Furthermore, chain connectivity could extend the influence of the interaction out several tens of nanometers from the surface [14]. The rubbery layer lies above the glassy layer, which is mobile above $T_{g}$.

According to the elastic theory [11], the local stress component tangential to the surface is responsible for the lateral flow of the film thickness. If we denote this term as $\sigma_{\tau}(x), p(x, t)$ is given by

$$
p=\frac{A}{6 \pi h^{3}}-\gamma \frac{\partial^{2} h}{\partial x^{2}}+\frac{\sigma_{\tau}^{2}}{2 E},
$$

where $A$ is the Hamaker constant for the van der Waals interaction of the film with the surrounding media, and $\gamma$ and $E$ are the surface tension and the Young's modulus of the film, respectively. Equation (2) is valid for initial perturbation, or $\partial h / \partial x \ll 1$.

When the surface is subjected to a plane stress loading $\sigma_{0}$, a linearized perturbation solution for the surface stress distribution gives [17]

$$
\sigma_{\tau}^{2}=\sigma_{0}^{2}(1-4 \varepsilon q \cos (q x)),
$$

where $\varepsilon$ is the perturbation amplitude and $q$ is the wave vector. Inserting Eq. (2) into Eq. (1) and keeping only terms linear in the amplitude of the fluctuations gives

$$
-C^{-1} \frac{\partial h}{\partial t}=\frac{A}{2 \pi h_{0}^{4}} \frac{\partial^{2} h}{\partial x^{2}}-\frac{1}{2 E} \frac{\partial^{2} \sigma_{\tau}^{2}}{\partial x^{2}}+\gamma \frac{\partial^{4} h}{\partial x^{4}} .
$$

We consider a small fluctuation of the film thickness about an initially uniform height $h_{0}$ :

$$
h(x, t)=h_{0}+\varepsilon \cos (q x) \exp (t / \tau) .
$$

Then, Eq. (5) yields the growth rate, $\tau^{-1}(q)$, of the mode with wave number $q$,

$$
(C \tau)^{-1}=\frac{A}{2 \pi h_{0}^{4}} q^{2}+\frac{2 \sigma_{0}^{2}}{E} q^{3}-\gamma q^{4}
$$

from which the mode selection behavior is derived. It has been assumed that $\sigma_{\tau}^{2}$ has the same exponential growth rate as for $h$. As expected, the elastic term acts as a destabilizing factor in the dewetting, which effectively increases the growth rate, $\tau^{-1}(q)$, compared with the stress-free film. For the wave number that grows the fastest, $q_{\max }$, we immediately obtain from Eq. (6)

$$
q_{\max }^{2}-\frac{3 \sigma_{0}^{2}}{2 E \gamma} q_{\max }-\frac{A}{4 \pi \gamma h_{0}^{4}}=0 .
$$

To compare the relative magnitude of the dispersion term with the stress term, we define two reciprocal lengths, $a_{1}$ and $a_{2}$ :

$$
a_{1}=\left(\frac{A}{4 \pi \gamma h_{0}^{4}}\right)^{1 / 2}, \quad a_{2}=\frac{3 \sigma_{0}^{2}}{2 E \gamma} .
$$

Then the solution of Eq. (8) is

$$
\frac{q_{\max }}{a_{1}}=\frac{a_{2}}{2 a_{1}}+\sqrt{1+\left(\frac{a_{2}}{2 a_{1}}\right)^{2}} .
$$

The thermal stress accumulated due to the temperature rise during annealing can be expressed as [18]

$$
\sigma_{0}=\int_{T_{0}}^{T} \frac{E}{1-\nu}\left(\alpha_{f}-\alpha_{s}\right) d T
$$

where $T_{0}$ is the stress-free temperature, $T$ is the temperature to which the film is heated, $\nu$ is Poisson's ratio, and $\alpha_{s}$ and $\alpha_{f}$ are the thermal expansion coefficients of the substrate and the film, respectively. With the film consisting of glassy and rubbery layers as shown in Fig. 1, the Young's modulus above $T_{g}$ can be written as

$$
E_{T>T_{g}}=\frac{h_{g}}{h_{0}} E^{g}+\left(1-\frac{h_{g}}{h_{0}}\right) E^{r},
$$

where $E^{g}$ and $E^{r}$ are Young's moduli for glassy and rubbery states, respectively, and $h_{g}$ is the thickness of the glassy layer, which should depend on the degree of confinement. The, Eq. (10) gives

$$
\begin{aligned}
\sigma_{0} & =\frac{\alpha_{f}-\alpha_{s}}{1-\nu}\left[\int_{T_{0}}^{T_{g}} E^{g} d T+\left(\frac{h_{g}}{h_{0}}\right) \int_{T_{g}}^{T} E^{g} d T+\left(1-\frac{h_{g}}{h_{0}}\right) \int_{T_{0}}^{T_{g}} E^{r} d T\right] \\
& \approx\left(\frac{T_{g}-T_{0}}{T-T_{0}}+\frac{h_{g}}{h_{0}} \frac{T-T_{g}}{T-T_{0}}\right)\left(\frac{E_{\mathrm{eff}}}{1-\nu}\right)\left(\alpha_{f}-\alpha_{s}\right)\left(T-T_{0}\right),
\end{aligned}
$$


where $E_{\text {eff }}$ is the effective Young's modulus that can explain the weak temperature dependence of $E^{g}$. It is noted that $E^{g} \gg E^{r}$.

Two extreme cases are considered first for the mode selection dependence on the film thickness. In one extreme case of an initially stress-free film $\left(\sigma_{0}=0\right), a_{2}$ is zero and $q_{\max }$ is proportional to $h_{0}^{-2}$, the power index for the wavelength being 2 . In the other extreme in which the stress term dominates over the dispersion term such that $\left(a_{2} / 2 a_{2}\right) \gg 1, q_{\max }$ is equal to $a_{2}$ and becomes independent of the thickness. In this extreme case, the power index for the thickness dependence becomes zero. When both stress and dispersion forces come into play, therefore, the power index can range anywhere from 0 to 2 .

Figure 2 shows the effect of film thickness on $q_{\max }$ for several values of $\left(E_{\text {eff }}^{2} / A \gamma\right)^{1 / 2}$ that determines the magnitude of $a_{2} / a_{1}$ in Eq. (9). Typical parameter values for glassy polymers such as PS are used in the figure, for which $\nu=0.3$ [19], $h_{g}=20 \mathrm{~nm} \mathrm{[14],} T_{g}=$ $100{ }^{\circ} \mathrm{C}, T_{0}=25^{\circ} \mathrm{C}, T=125^{\circ} \mathrm{C}$, and $\left(\alpha_{f}-\alpha_{s}\right)(T-$ $\left.T_{0}\right)=10^{-3}$ [19]. For $\left(E_{\text {eff }}^{2} / A \gamma\right)^{1 / 2}$ smaller than about $1 \mathrm{~nm}^{-2}, q_{\max } / a_{1}$ is about 1 throughout the thickness range, which is the result of the conventional capillary wave model. However, the value deviates from 1 as $\left(E_{\text {eff }}^{2} / A \gamma\right)^{1 / 2}$ increases and this effect is more pronounced for thicker film. The effect of thermal stress is not restricted to the glassy polymer but can equally be applicable to the elastomer. In the case of elastomers such as polydimethylsiloxane, the Young's modulus is on the order of MPa [19], which drastically decreases the value of $\left(E_{\mathrm{eff}}^{2} / A \gamma\right)^{1 / 2}$. However, thermal expansion coefficient

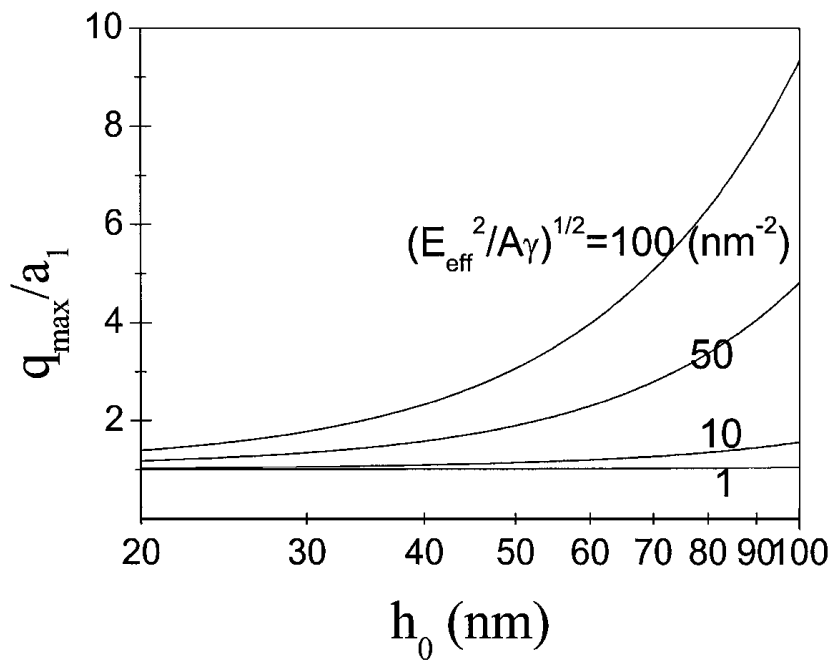

FIG. 2. Effect of thermal stress on the selection of dominant wave mode as a function of film thickness in the range between 20 and $100 \mathrm{~nm}$. As shown in the figure, the value of $q_{\max } / a_{1}$ deviates from 1 as $\left(E_{\text {eff }}^{2} / A \gamma\right)^{1 / 2}$ increases, especially for thicker films. Typical parameter values for glassy polymers such as PS are used in the figure, for which $\nu=0.3, h_{g}=20 \mathrm{~nm}$, $T_{g}=100{ }^{\circ} \mathrm{C}, \quad T_{0}=25^{\circ} \mathrm{C}, \quad T=125^{\circ} \mathrm{C}$, and $\left(\alpha_{f}-\alpha_{s}\right) \times$ $\left(T-T_{0}\right)=10^{-3}$. of the elastomer is much larger $\left(\alpha_{f} \sim 10^{-4}\right)$ [19] than that of the glassy polymer such that the overall behavior is maintained.

In a similar way, deviation from the capillary wave model can also be explained for metal films in dewetting experiments [10]. In this case, Young's modulus may sharply decrease at the phase transition, leading to a smaller value compared with the polymer. However, the fact that most dewetting experiments of metal films are carried out using rapid thermal processing implies that the residual thermal stress may not be completely annihilated during the melting [20]. Furthermore, the absolute value of the Young's modulus of metal is significantly larger than that of polymer by 3 orders of magnitude [18], which is another possibility for elastic contribution to dewetting.

To visualize the effect of residual thermal stress on the mode selection, we determined the power index in the thickness range between 20 and $100 \mathrm{~nm}$. We used typical parameter values for the glassy polymer: $A=10^{-19} \mathrm{~J}$ [21] and $\gamma=10^{-2} \mathrm{~J} / \mathrm{m}^{2}$ [21]. The slope shown in Fig. 3 is obtained from the regression that best fits the data points calculated from Eq. (9). As shown in the figure, the power index is strongly dependent on the effective Young's modulus, especially around $10^{9} \mathrm{~Pa}$. Considering that the Young's modulus of a typical glassy polymer is on the order of $10^{9} \mathrm{~Pa}$, the behavior in Fig. 3 can readily be realized in experiments. On the other hand, the thermal stress becomes negligible for a low molecular weight polymer, for which the effective Young's modulus is much smaller than $10^{8} \mathrm{~Pa}$.

In summary, we present a general model that can explain the dynamic instability of thin polymer films under a

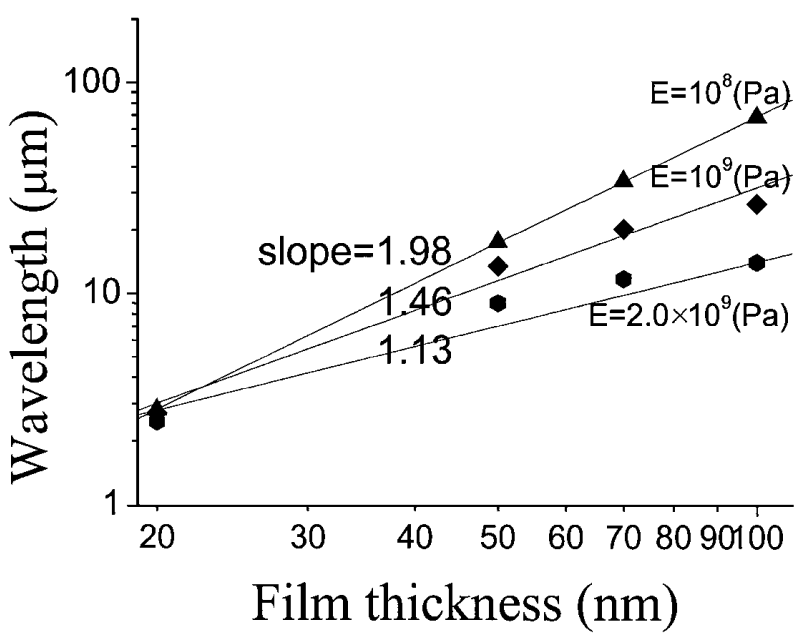

FIG. 3. Determination of the power index from the regression of data points calculated from Eq. (9) in the thickness range between 20 and $100 \mathrm{~nm}$. The power index decreases to values smaller than 2 as the effective Young's modulus increases. Typical parameter values for glassy polymers such as PS are used in the figure, for which $\nu=0.3, h_{g}=20 \mathrm{~nm}$, $\left(\alpha_{f}-\alpha_{s}\right)\left(T-T_{0}\right)=10^{-3}, T_{g}=100{ }^{\circ} \mathrm{C}, T_{0}=25^{\circ} \mathrm{C}, T=$ $125^{\circ} \mathrm{C}, A=10^{-19} \mathrm{~J}$, and $\gamma=10^{-2} \mathrm{~J} / \mathrm{m}^{2}$. 
highly confined state. The analysis shows that the thermal stress accumulated due to the temperature rise becomes pronounced when there is a special interaction and the molecular weight is high. Our results also clearly show that the power index can have a value lower than 2 depending on the relative magnitude of dispersion force and thermal stress.

*To whom correspondence should be addressed. Email address: honghlee@plaza.snu.ac.kr

[1] M. Schick, in Liquids at Interfaces, Proceedings of the Les Houches Summer School, Session XLVIII, edited by J. Charvolin et al. (Elsevier, Amsterdam, 1990).

[2] S. Garoff, E. B. Sirota, S. K. Sinha, and H. B. Stanley, J. Chem. Phys. 90, 7505 (1989).

[3] F. Brochard-Wyart and J. Daillant, Can. J. Phys. 68, 1084 (1990).

[4] E. A. L. Mol, G. C. L. Wong, J.-M. Petit, F. Rieutord, and W. H. de Jeu, Phys. Rev. Lett. 79, 3439 (1997).

[5] S. Herminghaus et al., Science 282, 916 (1998).

[6] G. Reiter, Phys. Rev. Lett. 68, 75 (1992).

[7] R. Xie, A. Karim, J. F. Douglas, C. C. Han, and R. A. Weiss, Phys. Rev. Lett. 81, 1251 (1998).

[8] J. Wang et al., Phys. Rev. Lett. 83, 564 (1999).

[9] M. Tolan et al., Phys. Rev. Lett. 81, 2731 (1998).
[10] J. Bischof, D. Scherer, S. Herminghous, and P. Leiderer, Phys. Rev. Lett. 77, 1536 (1996).

[11] R. J. Asaro and W. A. Tiller, Metall. Trans. 3, 1789 (1972); M. A. Grinfeld, Dokl. Akad. Nauk. USSR 283, 1139 (1985); D. J. Srolovitz, Acta Metall. 37, 621 (1989); B. J. Spencer, P. W. Voorhees, and S. H. Davis, J. Appl. Phys. 73, 4955 (1993).

[12] E. Schäffer, T. Thurn-Albrecht, T.P. Russell, and U. Steiner, Nature (London) 403, 874 (2000).

[13] A. Ghatak, M. K. Chaudhury, V. Shenoy, and A. Sharma, Phys. Rev. Lett. 85, 4329 (2000); K. R. Shull, C. M. Flanigan, and A. J. Crosby, Phys. Rev. Lett. 84, 3057 (2000).

[14] W. E. Wallace, J. H. van Zanten, and W. L. Wu, Phys. Rev. E 52, R3329 (1995); J. H. van Zanten, W. E. Wallace, and W. L. Wu, Phys. Rev. E 53, R2053 (1996).

[15] J.D. Ferry, Viscoelastic Properties of Polymers (John Wiley, New York, 1980), 3rd ed.

[16] S. Herminghaus, Phys. Rev. Lett. 83, 2359 (1999).

[17] H. Gao, J. Mech. Phys. Solids 42, 741 (1994).

[18] S. P. Timoshenko and J. N. Goodier, Theory of Elasticity (McGraw-Hill, New York, 1970), 3rd ed.

[19] Polymer Handbook, edited by J. Brandrup and E. H. Immergut (John Wiley, New York, 1989), 3rd ed.

[20] J. Siegel, K. Ettrich, E. Welsch, and E. Matthias, Appl. Phys. A 84, 213 (1997).

[21] J. Isaellachivili, Intramolecular Surfaces Forces (Academic Press, New York, 1992), 2nd ed. 